Revue d'histoire de l'Amérique française

ZHA REVUE D.HISTOIRE DE L'AMERIQUE FRANÇAISE

\title{
La standardisation des études médicales et la consolidation de la profession dans la deuxième moitié $\mathrm{du}^{\mathrm{XIX}}{ }^{\mathrm{e}}$ siècle
}

\section{Jacques Bernier}

Volume 37, numéro 1, juin 1983

URI : https://id.erudit.org/iderudit/304124ar

DOI : https://doi.org/10.7202/304124ar

Aller au sommaire du numéro

Éditeur(s)

Institut d'histoire de l'Amérique française

ISSN

0035-2357 (imprimé)

1492-1383 (numérique)

Découvrir la revue

Citer cet article

Bernier, J. (1983). La standardisation des études médicales et la consolidation de la profession dans la deuxième moitié du $\mathrm{XIX}^{\mathrm{e}}$ siècle. Revue d'histoire de l'Amérique française, 37(1), 51-65. https://doi.org/10.7202/304124ar d'utilisation que vous pouvez consulter en ligne. 


\title{
LA STANDARDISATION DES ÉTUDES MÉDICALES ET LA CONSOLIDATION DE LA PROFESSION DANS LA DEUXIÈME MOITIÉ DU XIX SIĖCLE
}

\author{
JACQUES BERNIER \\ Université Laval
}

\begin{abstract}
In a fragmented and harshly competitive market, economic survival was linked to the excessive number of practitionners. Regular physicians, moreover, faced the problem of enforcing discipline within their own rank if they wanted to maintain their precariorus distinctiveness. The supply of medical services had to be both reduced and standardized.*
\end{abstract}

Ce texte s'inscrit dans le cadre d'une recherche sur le développement de la profession médicale au Québec au XIX ${ }^{\mathrm{e}}$ siècle. Un article, paru en 1980, a montré les efforts faits par certains médecins civils de Québec et de Montréal, entre 1810 et 1830, pour réformer la médecine et la profession médicale dans la colonie ${ }^{1}$. Un autre, paru l'année suivante, décrivait leurs démarches, entre 1831 et 1847 , afin de regrouper le corps médical et de l'organiser en corporation autonome ${ }^{2}$.Ces recherches nous ont permis de constater que, au milieu du XIX ${ }^{\mathrm{e}}$ siècle, le processus de professionnalisation de la médecine est déjà bien engagé. On a pu remarquer, par exemple, dans les villes, l'existence de sociétés médicales qui regroupaient, sur une base volontaire, plusieurs praticiens. En 1847, une loi a reconnu aux chirurgiens et médecins de la province le droit de se constituer en corporation professionnelle. Pour promouvoir les études médicales, une faculté et deux écoles de médecine furent créées dont la fréquentation était devenue obligatoire à tous les futurs praticiens. Enfin, des règlements plus sévères avaient été institués pour empêcher l'exercice illégal de la médecine.

* M.S. Larson, The Rise of Professionalism, a Sociological Analysis (Berkeley, University of California Press, 1977), 131

1 Jacques Bernier, «François Blanchet et le mouvement réformiste en médecine au début du XIX ${ }^{\mathrm{e}}$ siècle», Revue d' histoire de l'Amérique française, 34, 2 (septembre 1980): 223-244.

2 Jacques Bernier, «Vers un nouvel ordre médical: les origines de la Corporation des médecins et chirurgiens du Québec», Recherches sociographiques, XXII, 3 (septembre-décembre 1981): 307-330. 
Plus récemment, notre attention s'est portée sur la fin du XIX siècle. Un phénomène important à se produire durant cette période est que le corps médical a réussi à consolider et à étendre considérablement les pouvoirs qui lui avaient été conférés en 1847. Cette consolidation s'est faite de trois manières principales. La première a trait à la standardisation des études médicales dans les institutions nouvellement créées, et à l'uniformisation des diplômes et des permis à la fin des études. La deuxième concerne une série de mesures internes visant à régulariser la vie du groupe et en rapprocher les membres. La troisième, quant à elle, est reliée au contexte des luttes menées par le corps médical, contre des groupes concurrents et face aux autorités politiques, pour élargir son domaine d'intervention et renforcer ses droits d'autonomie et d'exercice exclusif de la médecine.

Le présent texte porte sur le premier de ces trois volets. Il vise à expliquer le contexte et les facteurs qui furent à l'origine de la standardisation des études médicales à la fin du XIX ${ }^{\mathrm{e}}$ siècle. En d'autres mots, on y étudie quand, comment et pourquoi on est arrivé à imposer le cours classique à l'entrée des études, un cursus uniforme pour toutes les institutions d'enseignement, et les mêmes examens pour l'admission à la pratique. Cette standardisation, comme nous le verrons, ne se fera pas sans difficultés. En fait, le Collège des médecins et chirurgiens qui, pour une large part, est l'acteur principal de ce projet aura du mal à affirmer son autorité auprès des facultés de médecine qui cherchent à sauvegarder leur autonomie et leurs privilèges.

Son intervention, le Collège la justifie par les droits qui lui ont été conférés par la loi de 1847 , laquelle, à l'article X, lui reconnaît «le pouvoir de régler l'étude de la médecine, de la chirurgie, de l'art obstétrique et de la pharmacie, en établissant des règlements quant à la qualification préliminaire, la durée des études, le cours à suivre, et l'âge de l'aspirant» ${ }^{3}$. Quant aux écoles, après être devenues des facultés de médecine dans les années 1860-1870, elles invoquent le principe de la liberté de l'enseignement universitaire et une coutume qui remonte à la fondation de la faculté de médecine de McGill pour prétendre ne pas être liées par les interprétations que le Collège fait des règlements de la loi de 1847 relatifs aux études médicales. Ces règlements, elles se sentent donc libres de les appliquer comme elles le veulent. De là naîtra le conflit entre, d'une part, le Collège qui prétend représenter les intérêts de l'ensemble des praticiens, et d'autre part, les facultés qui ne veulent pas perdre leurs privilèges.

Mais avant de décrire les procédures qui vont être entreprises par le Collège, en vue d'uniformiser les études dans les écoles de médecine de la province à la fin du $\mathrm{XIX}^{\mathrm{e}}$ siècle, il est nécessaire de faire un bref

3 Statuts du Bas-Canada, 10-11 Victoria, chap. 26, 1847. 
rappel sur le rôle des écoles médicales à cette époque, car c'est bien avec elles, et ce avant l'intervention du Collège, que les bases d'une formation uniforme en médecine ont été posées dans la province.

\section{L'établissement des écoles et des facultés de médecine}

Il existe une "préhistoire» des écoles de médecine qui se situe en gros au début du XIX ${ }^{\mathrm{e}}$ siècle. Cette phase se caractérise par l'existence de cours de médecine donnés par des praticiens dans les hôpitaux, des dispensaires et même à domicile. Ainsi est-il fréquent de trouver dans les journaux de l'époque des annonces comme celle-ci: «Les cours du Docteur Painchaud sur la médecine et sur les accouchements commenceront le 5 mai prochain à l'Hôpital de Marine. ${ }^{4}$ Ces cours ne sont pas obligatoires; ils ne sont pas donnés sous l'égide d'une institution; leur contenu et leur durée ne sont pas réglementés et ils ne s'inscrivent pas dans le cadre de programmes. D'ailleurs, il n'y a ordinairement pas de contrôle à la fin de ces cours. En fait, ils relèvent de la seule autorité du médecin qui les donne. Lui seul en est le responsable et il n'a de compte à rendre à personne. Les rémunérations qu'il retire proviennent exclusivement des étudiants inscrits. Il est difficile d'établir le nombre des cours qui étaient ainsi dispensés pendant une année, dans une ville comme Québec par exemple, parce que plusieurs personnes en donnaient. On sait par contre que, durant la décennie 1830-40, à l'hôpital de Marine, presque toutes les matières étaient enseignées (anatomie, instituts de médecine, pathologie, materia medica, chirurgie, accouchements et maladies des femmes, pratique de la médecine).

Ce qui est nouveau avec la loi de 1847 , c'est que ces cours deviennent obligatoires, qu'ils doivent être suivis dans des institutions reconnues et que ceux qui les suivent sont astreints à des examens. Au Québec comme aux États-Unis et en Ontario, le nombre de ces écoles s'accrut rapidement. En fait, six écoles différentes virent le jour entre 1824 et 1878: The Montreal Medical Institution en 1824 (qui deviendra la Faculté de médecine de McGill en 1829), l'École de médecine et de chirurgie de Montréal (1845), l'École de médecine de Québec (incorporée en 1845 et affiliée à Laval en 1854), St-Lawrence (1851), Bishop's (1871), et la succursale de Laval à Montréal (1878) ${ }^{5}$. Il n'y a pas lieu de revenir ici sur l'histoire de chacune de ces institutions puisqu'on la trouve déjà dans d'autres ouvrages, notamment dans Abbott, Heagerty, Boissonneault, et Lavallée ${ }^{6}$. Par contre, il peut être utile de rap-

4 Le Canadien, 7 avril 1847.

Sur le nombre de professeurs, d'élèves et de volumes dans ces trois premières institutions, voir le Rapport du surintendant de l'éducation dans le Bas-Canada pour l'année 1857 (Toronto, J. Lovell, 1858), 48-49,.

6 M.E. Abbott, History of Medecine in the Province of Québec (Montréal, McGill University, 1931). - J.J. Heagerthy, Four Centuries of Medical History in Canada (Toronto, Macmillan, 1928), II: 55-128. - C.-M. Boissonneault, Histoire de la Faculté de médecine de Laval (Québec, Presses de l'Université Laval, 1953). - A. Lavallée, Québec contre Montréal, la querelle universitaire, 1876-1891 (Montréal, Presses de l’Université de Montréal, 1974). 
peler certains changements qui ont été apportés dès la mise en place de ces écoles. Cette nouvelle formule a d'abord permis de fournir une formation plus complète, plus poussée et mieux encadrée non seulement parce que les agents de formation étaient plus nombreux mais parce que les expériences pédagogiques l'étaient également. En plus de recevoir des cours théoriques, l'étudiant pouvait désormais bénéficier de la présence d'un musée d'anatomie, d'une bibliothèque et de séances de dissection. Il faisait aussi des stages pratiques dans les hôpitaux et dispensaires affiliés à son institution d'enseignement et pouvait ainsi bénéficier d'une plus vaste expérience clinique. Cette formule, par ailleurs, a contribué à unifier l'enseignement de la médecine dans la province. Avec elles, l'éducation devint beaucoup plus formelle. Il ne s'agissait plus d'une relation entre un étudiant et son maître comme dans l'apprentissage, ou d'une relation libre comme dans la phase préinstitutionnelle. La fréquentation d'une école était maintenant un fait obligatoire. Cette formation se faisait désormais dans un lieu précis et par un groupe choisi de professeurs. Ces professeurs avaient désormais un rôle tout à fait central puisque c'étaient eux, et eux seuls, qui d'année en année formaient les futurs praticiens. Ainsi on assistait non seulement à une centralisation des lieux de formation, mais des agents mêmes de cette formation. Ceci dit, les règlements variaient considérablement d'une institution à une autre. Les démarches du Collège en vue de les uniformiser allaient toutefois poser certains problèmes.

\section{L'uniformisation des conditions d'entrée aux études}

Le problème des conditions d'admission aux études était important. Il se formulait ainsi: comment faire en sorte que les institutions d'enseignement en arrivent à se soumettre aux mêmes procédures et aux mêmes exigences quant à l'admission aux études?

Il ne s'agissait pas là d'un problème propre à cette période. En fait, il en avait été question souvent au début du siècle. C'est d'ailleurs à la suite de pressions en ce sens que l'article VIII de la loi de 1847 était venu préciser à cet égard que personne ne serait admis à l'étude de la médecine, de la chirurgie et de l'art obstétrique avant d'avoir obtenu un certificat du Bureau provincial de médecine. Contrairement à la loi de 1831, les exigences requises étaient maintenant définies. C'était précisément celles que les Bureaux des examinateurs de Québec et de Montréal avaient proposées durant les années 1840, soit: un certificat de bonnes moeurs, une connaissance suffisante du latin, de l'histoire, de la géographie, des mathématiques, de la philosophie de la nature et une bonne connaissance des langues française et anglaise. En somme, il s'agissait des éléments de base de la formation classique au Canada. Une clause exemptait cependant de ces examens les écoles affiliées à une université ayant une charte britannique ${ }^{7}$. Celles-ci étaient libres

\footnotetext{
7 Statuts du Bas-Canada, 10-11 Victoria, chap. 26, 1847 (art. VII).
} 
d'admettre qui elles voulaient, sans obtenir l'assentiment des représentants du Collège. Cette clause fut à l'origine de nombreuses querelles. Les écoles non affiliées étaient évidemment frustrées de ne pas bénéficier d'un tel privilège. L'École de médecine et de chirurgie, qui ne trouva à s'affilier qu'en 1866 à l'université Victoria, fut celle qui revint le plus souvent sur ce sujet, en demandant que tous les aspirants à la médecine soient soumis aux examens du Bureau; faute de quoi le même privilège devait être accordé aux écoles. Cette restriction agaçait aussi le Collège dont l'autorité en ce domaine se trouvait de plus en plus diminuée. C'est ainsi qu'il perdit son droit de regard sur les étudiants de Laval en 1852, sur ceux de l'École de médecine et de chirurgie en 1866, et finalement sur ceux de Bishop's en 1871. Enfin d'autres conflits naissaient du fait que certaines institutions se montraient plus libérales que d'autres dans l'application des critères.

Le premier projet de réforme important et structuré pour trouver une solution à ce problème fut présenté en 1868 , à l'occasion de la deuxième rencontre annuelle de l'Association médicale du Canada. Il était l'oeuvre du comité chargé de faire rapport sur les examens préliminaires à l'étude de la médecine. Le but poursuivi était de définir des critères applicables à travers tout le Dominion. Le modèle proposé était celui de la loi médicale anglaise de 1858. Le président de ce comité était le Dr Rowand de Montréal, qui fut pendant longtemps gouverneur du Collège. Les propositions principales de son comité furent les suivantes: a) les examens d'entrée aux études médicales devraient être faits par un comité de professeurs autres que ceux des facultés ou écoles de médecine; b) ces examens devraient porter sur les langues française et anglaise, l'arithmétique, les règles élémentaires et fractions décimales, l'algèbre, la géométrie, le latin (traduction et grammaire), la philosophie naturelle et la logique ${ }^{8}$. Rottot, qui enseignait à l'École de médecine et de chirurgie et qui était lui aussi gouverneur du Collège, soutint quelques années plus tard des points de vue semblables:

Il est important, disait-il, de priver les universités de faire l'examen classique des aspirants à l'étude de la médecine. L'expérience, en effet, nous a démontré que cet examen est loin d'être sérieux. Bien souvent même, on a permis aux élèves de commencer l'étude de la médecine, on leur a laissé compléter leur cours et on leur a fait subir leur examen classique quelques instants seulement avant leur examen médical. Ce privilège, en donnant aux universités la faculté de se faire des élèves, laisse la profession tout-à-fait sans protection. Mais en donnant au corps médical seul le droit d'examiner les élèves sur leur éducation préliminaire, et de les admettre à l'étude de la médecine, nous nous assurons la

\footnotetext{
${ }^{8}$ Le Journal de Québec, 5 septembre 1868.
} 
protection la plus simple possible sans mettre le moindre obstacle à la prospérité des universités. ${ }^{9}$

Un des éditorialistes de l'Union médicale du Canada laissa un témoignage semblable en 1875: «La profession médicale (le Collège) doit posséder les pouvoirs nécessaires pour gérer ses propres affaires; à elle appartient de décider quels sont ceux qu'elle admettra dans son sein et les qualifications qu'elle exigera des aspirants.» ${ }^{10}$

En fait, cette recherche d'uniformisation des conditions et des procédures d'admission n'avait pas pour seul but de rehausser le niveau des études. Cette démarche était motivée aussi par un autre objectif, celui de freiner le recrutement. On trouvait que trop de jeunes s'orientaient vers la médecine et parmi eux trop d'incompétents. D'Orsonnens, déjà en 1862, avait soulevé le problème ainsi: «Il faut éviter, disait-il que les professions ne soient regardées à l'instar du plus vil métier.» ${ }^{11}$ Brousseau, avant l'adoption de la loi de 1876, tenait des propos semblables: «...trop de jeunes s'engagent dans la carrière sans avoir consulté leurs forces, il faut bien faire quelque chose, toutes les routes sont emcombrées, va pour la médecine. ${ }^{12}$

En 1876, faisant suite à ces pressions et à celles du Collège et de l'Union médicale, une nouvelle loi fut adoptée par la Chambre. Des modifications majeures étaient apportées, qui donnaient plus d'autorité au Collège. Une clause disait que les aspirants, pour être admis en médecine, devraient passer un examen devant un jury de quatre personnes nommées pour quatre ans par le Collège. Ces personnes devraient être engagées dans l'oeuvre de l'éducation, mais non rattachées à des écoles ou facultés de médecine. Les matières à examen étaient maintenant plus nombreuses et comprenaient l'anglais, le français, le latin, la géographie, l'histoire, l'algèbre, la géométrie, les belles-lettres, et l'un des sujets suivants: le grec, la physique ou la philosophie. Le certificat de bonnes moeurs restait obligatoire ${ }^{13}$. Quelques années plus tard, J.-P. Rottot, devenu président du Collège, commentait ainsi dans son rapport triennal les bienfaits de cette loi: «Nous avons maintenant les aspirants à l'étude de la médecine entièrement sous notre contrôle [...], entre 1877 et 1880 sur les 127 demandes d'admission, 45 ont été refusées ${ }^{14}$, soit $35,5 \%$. Entre 1880 et 1884 cette proportion monta à $40 \%$, et même davantage ${ }^{15}$.

\footnotetext{
9 Cité par A. LeSage, Le Centenaire du Collège des médecins et chirurgiens de la province de Québec (Montréal. s.l.n.e., 1947), 25.

${ }_{10}$ L'Union médicale du Canada, IV, 11 (novembre 1875): 521.

11 A.S.Q., U 102, BD; 27 novembre 1862.

12 L'Union médicale du Canada, III, 11 (novembre 1874): 481.

13 Statuts de la province de Québec, 40 Victoria, chap. 26, 1876 (art. IX).

14 L'Union médicale du Canada, IX, 7 (juillet 1880): 388-390.

15 H. Provost, Historique de la Faculté des arts de l'Université Laval (Université Laval, thèse de maîtrise ès arts en histoire, 1952), 105.
} 
La loi de 1876 fut modifiée en 1890 . Cette année-là une autre loi, connue sous le nom de la loi Hall, fut sanctionnée par la Chambre. Cette loi, qui fut en fait proposée par les universités, visait à inciter les candidats aux professions médicale, légale et notariale à faire leurs études classiques en entier ${ }^{16}$. Elle reconnaissait aux bacheliers ès arts, ès lettres et ès sciences, le droit d'être admis à l'étude d'une profession sans avoir à se présenter devant le Collège ${ }^{17}$. Les autres, par contre, devaient obtenir, comme on le faisait depuis 1876, un certificat de capacité du Bureau provincial de médecine. Le Collège était d'accord avec l'idée de prolonger les études mais il voyait d'un mauvais oeil le rôle que joueraient à nouveau les universités dans l'admission aux études.

L'uniformisation des règles d'accès aux études s'est donc faite relativement tard. Le véritable mouvement a démarré après la Confédération, dans le contexte des débats en vue d'uniformiser les règlements relatifs à la vie médicale au Canada. Le Collège profita de ce contexte et des arguments invoqués en vue de réformer les conditions d'accès à l'étude de la médecine dans le Dominion pour justifier son propre projet au Québec. Mais nous verrons plus loin qu'il se dissocia de l'idée de la mise en place d'un organisme pan-canadien en invoquant le caractère particulier du Québec et le principe de l'autonomie de la province en matière d'éducation et de santé.

\section{L'uniformisation des études}

Le problème de la standardisation des études médicales ne se posait pas tant au plan du programme, c'est-à-dire des matières obligatoires, qu'à celui de la durée des études et de l'assiduité des étudiants durant leur formation. À notre connaissance, il n'y eut pas de véritable débat sur les programmes d'études durant cette période.

La loi de 1847 était venue fixer pour la première fois le cursus médical dans la province. Désormais la seule formation par apprentissage ne suffirait plus; il faudrait en plus fréquenter une école ou une faculté de médecine. La loi de 1847 fixa le nombre des cours obligatoires à 15 , soit deux cours d'anatomie générale et de physiologie, deux cours d'anatomie pratique, deux cours de chirurgie, deux cours de pratique de la médecine, deux cours d'obstétrique, deux cours de chimie, un cours de matière médicale et de pharmacie, un cours des instituts de la médecine de six mois, un cours de jurisprudence médicale de trois mois et un cours de botanique de trois mois quand cela était possible. La formation pratique consistait à: 1) avoir été intégré à la pratique générale d'un hôpital contenant au moins 50 lits pendant une année ou l'équivalent; 2) avoir suivi deux cours de médecine clinique et deux

\footnotetext{
16 Ibid., 104.

17 Statuts de la Province de Québec, 53 Victoria, chap. 45, 1890.
} 
cours de chirurgie clinique de trois mois chacun ${ }^{18}$. Il s'agissait là d'exigences minimales. Certaines difficultés apparurent cependant à cause de la liberté qu'avaient les institutions d'organiser l'enseignement de ces cours comme elles l'entendaient. Au début des années 1860, l'Université Laval voulait, dit-on, que ses étudiants reçoivent une formation semblable à celle des universités françaises. Ainsi, elle «donnait en chaque matière plus de leçons que McGill et l'École de médecine et de chirurgie de Montréal» ${ }^{19}$ et exigeait quatre années de neuf mois et demi chacune $^{20}$. Alors que les autres institutions n'émettaient qu'un seul diplôme, l'Université Laval en donnait trois. Le premier des trois, le baccalauréat en médecine, s'obtenait après neuf sessions et après l'obtention de la note bien ou très bien dans toutes les matières. Pour obtenir la licence (ou maîtrise) il fallait subir deux épreuves écrites et une épreuve orale d'au moins six heures chacune. Le doctorat pouvait être accordé après l'obtention de la licence et la soutenance d'une thèse durant au moins trois heures ${ }^{21}$. Les épreuves que l'Université Laval demandait aux aspirants au doctorat étaient trop nombreuses comparativement à celles des autres institutions semblables au Québec, de sorte qu'en 1865 elle se résigna à changer ses règlements et décida du même coup de renoncer à la thèse. Elle reconnaissait désormais comme docteurs ceux qui avaient conservé les notes bien ou très bien aux examens de la licence. Les autres recevaient le diplôme de licenciés en médecine de l'Université 22 .

De son côté, l'École de médecine et de chirurgie, qui n'avait pas de visées aussi hautes, s'en tenait aux règlements visant le contenu des études, mais se montrait moins exigeante que les autres institutions quant à la durée des études. Suivant sur ce point l'exemple de certaines universités américaines et canadiennes (comme Queen's) ${ }^{23}$, elle n'exigeait que deux années de résidence. Comme le disait lui-même le directeur de l'École en 1862, «lorsque les élèves ont étudié à notre école deux sessions de six mois chacune et qu'ils ont étudié avec un patron un temps suffisant pour compléter avec ces différentes sessions quatre années d'études, ils ont le droit de passer devant le bureau l'examen qui leur permet d'obtenir leur permis» ${ }^{24}$.

La situation était encore différente à McGill. Les études duraient en principe quatre années, mais trois seulement devaient être passées

18 Statuts du Bas-Canada, 10-11 Victoria, chap. 26, 1847 (art. XII).

19 A. Maheux, «Le centenaire de la Faculté de médecine de l'Université Laval», L'information médicale et paramédicale (3 juin 1952); Le Journal de Québec, 11 septembre 1862.

20 A.S.Q., Université 101, C F.

21 Annuaire de l'Université Laval pour l'année académique 1863-64: 28-29.

22 Le Journal de Québec, 5 octobre 1865 et 8 août 1865.

23 Medical Chronicle, VI, 5 (mai 1858): 225-227.

24 A.S.Q., Université 102, A Q. 
dans l'institution ${ }^{25}$. L'Université ne délivrait qu'un seul diplôme, le doctorat en médecine, qui s'obtenait après la rédaction d'une thèse ${ }^{26}$.

En somme, même si la loi prévoyait quatre années d'études, toutes les institutions n'interprétaient pas ce règlement de la même manière. Ce problème existait d'ailleurs aussi dans le reste du Canada. En 18671868 , lors des conventions annuelles de l'Association médicale du Canada, on souleva le problème en essayant d'établir des règles qui pourraient convenir aux quatre provinces. On recommanda le maintien des études à quatre années, mais en exigeant que, pour chacune, les cours s'étendissent sur neuf mois. On proposait aussi d'ajouter certains cours: chimie pratique, chimie théorique, anatomie pathologique, hygiène publique ${ }^{27}$.

Au Québec, au milieu des années 1870, lorsqu'il fut question de réformer les études médicales, on s'inspira de ce modèle et on arriva à un consensus assez rapidement. La clause 14 de la loi de 1876 obligeait désormais les élèves à étudier quatre années sans interruption comprenant chacune trois sessions de trois mois ${ }^{28}$. Quant aux cours obligatoires, on en ajoutait sept au bloc de 1847: chimie, hygiène, botanique, instituts de médecine, thérapeutique générale, pathologie générale et 25 leçons d'anatomie, de physiologie et de pathologie microscopiques. La période de stage dans un hôpital passait de un an à un an et demi. On précisait aussi qu'il fallait avoir assisté à six accouchements et manipulé des remèdes pendant six mois ${ }^{29}$. Enfin, avec la loi de 1876 , disparaissait une coutume centenaire au Québec et au Canada, la cléricature ou l'apprentissage auprès d'un confrère ${ }^{30}$. Les salles de cours et les salles d'hôpitaux devenaient les seuls lieux de formation reconnus.

Entre 1876 et la fin du siècle, une dizaine de lois relatives à la médecine ont été votées à la Chambre. Aucune d'elles ne vint réformer le cursus médical en profondeur. Contrairement à ce qu'on peut penser, ce ne sont pas les études médicales elles-mêmes qui font surtout l'objet de débats à l'époque mais bien des points secondaires comme l'entrée aux études, les permis et l'exercice illégal de la médecine. Il faudra attendre 1909 avant qu'une autre loi ne vienne changer en profondeur l'enseignement de la médecine dans la province. Le programme passa alors à cinq années et fit place, tardivement, aux nouvelles disciplines comme la bactériologie, la dermatologie, l'hygiène publique, la pédiatrie, etc.

\footnotetext{
25 Medical Chronicle, VI, 5 (mai 1858): 225-227.

C'est seulement au début du $\mathrm{XX}^{\mathrm{e}}$ siècle que la thèse cessa d'être obligatoire à McGill Le Journal de Québec, 5 septembre 1868.

Statuts de la Province de Québec, 40 Victoria, chap. 26, 1876, (art. XIV).

Chaque cours comprend 120 leçons.

30 S. Leblond, «La profession médicale sous l'Union, 1840-1867», Les Cahiers des Dix, 38 (1973): 199.
} 


\section{L'uniformisation des diplômes et permis}

La nécessité de détenir un permis pour pratiquer la médecine dans la province remonte à 1788 . Ces permis étaient accordés par deux organismes, les Bureaux d'examinateurs de Québec et de Montréal. En 1832 , on commença à déroger à ce principe lorsque McGill, qui venait d'être créée, obtint le droit de décerner des diplômes ad practicandum à cause de son statut d'université britannique ${ }^{31}$. La loi de 1847 ratifia ces deux pratiques, mais elle revint au principe d'un seul examinateur, qu'elle plaça sous l'autorité du Collège. Ce nouveau Bureau, selon la loi, devait se réunir deux fois par année pour faire subir les examens aux candidats. Le diplôme, par contre, était émis par le Gouverneur. C'est en 1849 que ce privilège revint au Collège. Les diplômés de McGill continuèrent toutefois d'être exemptés de cet examen à cause de l'article VII qui stipulait que les diplômés de toute université dans le domaine de sa Majesté avaient le droit à un tel certificat sans examen. Le Collège exerçait donc son droit de regard sur les diplômés de deux des trois institutions seulement, soit ceux de l'École de médecine et de chirurgie de Montréal et ceux de l'École de médecine de Québec. Entre 1852 et 1866, le Collège assista impuissant à l'érosion de ses pouvoirs en ce domaine à cause de l'affiliation de ces deux écoles à des universités ${ }^{32}$. La situation était telle qu'il ne restait plus au Collège que le pouvoir d'émettre des permis de pratique. J.-P. Rottot résuma ainsi la situation:

La loi de 1847 avait été rédigée dans le but de donner au collège le contrôle sur tout ce qui avait rapport à notre profession. Cependant le Bureau n'a jamais pu jouir de ce contrôle d'une manière absolue, parce qu'on avait mis dans la loi une clause qui donnait aux porteurs de diplômes universitaires le droit à la licence du Collège sans examen quant à leurs qualifications. Par cette clause le Collège perdit du coup tout pouvoir sur les étudiants en médecine de l'Université McGill et de l'Université Laval, et plus tard sur ceux de l'École de médecine et de chirurgie lors de son affiliation avec l'Université Victoria; de sorte qu'il fut obligé par la loi de donner sa licence à tous ceux qui se présentaient avec un diplôme, sans pouvoir s'assurer s'ils possédaient les qualifications et les connaissances requises. Le Bureau ne pouvait pas supporter une telle position; il fit, dès le début, tous ses efforts pour briser cet état de chose qu'il considérait tout à fait opposé aux intérêts généraux de la profession. Il s'agissait d'enlever à des institutions des privilèges importants qu'elles tenaient à conserver. ${ }^{33}$

${ }_{31}$ Voir à ce sujet, B. Tunis, «Medical Licensing in Lower Canada: The Dispute over Canada's First Medical Degree», Canadian Historical Review, LV, 4 (décembre 1974): 489-504; S. Leblond, op. cit.: 170.

32 L'École de médecine de Québec s'intégra à Laval en 1852 et celle de Montréal s'affilia à Victoria en 1866.

33 L'Union médicale du Canada, IX, 7 (juillet 1880): 388. 
La lutte menée par le Collège pour rétablir son autorité en ce domaine fut longue et difficile. Elle commença en fait dès 1847 et ne se termina qu'en 1909. Elle fut toujours orientée vers le même but: enlever à McGill, puis aux autres universités le pouvoir qu'elles avaient de décerner des diplômes ad practicandum (les détenteurs de ces diplômes universitaires avaient droit automatiquement à la licence du Collège). Quand W. Nelson accéda à la présidence du Collège en 1849, il essaya de faire modifier la loi pour que les diplômés de McGill subissent l'examen du Collège mais il n'y parvint pas ${ }^{34}$. Un des principaux porte-parole de ce courant d'opposition, dans les années 1850, fut le Dr Laterrière. C'est lui qui, en tant que député, souleva le problème à la Chambre. La situation, à ses yeux, était injuste non seulement parce que la loi admettait deux poids deux mesures, mais parce que les étudiants de McGill, tout en bénéficiant d'un statut privilégié, ne remplissaient pas la condition des quatre années d'études ininterrompues exigées par la loi ${ }^{35}$. Il s'en prenait également à l'article suivant lequel les diplômés des universités de Grande-Bretagne étaient autorisés à pratiquer au Canada; cela, prétendait-il, donnait lieu à des abus: «il est bien connu, écrivait-il, que des diplômes se vendent jusqu'à $£ 50$ à des individus qui n'avaient pratiquement aucune notion de médecine et de chirurgie. ${ }^{36}$ Mais ces critiques n'arrivèrent jamais à convaincre, sous l'Union, la majorité des députés, de sorte que son projet de loi fut finalement retiré.

Les débats sur ces questions reprirent en 1867 et 1868, lors des rencontres de l'Association médicale du Canada. En 1867, le Comité formé pour étudier le problème des permis au Canada fit remarquer qu'il n'y avait pas assez d'uniformité entre les institutions des provinces. Il proposa la mise sur pied d'un Conseil général d'examen pour le Dominion ${ }^{37}$. Mais au Québec, on ne fut pas d'accord avec ce projet, d'abord parce que les Canadiens français seraient minoritaires au sein de ce Conseil général, ensuite parce que ce serait la fin de l'autonomie du Collège, enfin parce que ce serait mettre l'éducation sous la tutelle d'une juridiction non provinciale ${ }^{38}$.

En 1868 un comité fut à nouveau formé pour étudier cette question, sous la présidence de G.W. Campbell de Montréal. La proposition qu'il soumit aux délégués laissait plus de liberté aux universités. Elle suggérait de former un Conseil médical qui aurait un droit de supervision sur la qualité de l'éducation dispensée dans les universités et sur les examens ${ }^{39}$. Au Québec, on se montra à nouveau réticent à l'idée de

\footnotetext{
34 S. Leblond, op. cit.: 185-186.

35 Le Canadien, 18 avril 1853.

Le Canadien, 18 avril 1853.

Morning Chronicle, 10 octobre 1867.

38 «Allons-nous, dit alors Rottot, nous laisser dépouiller de nos importantes et utiles prérogatives», A. Lesage, op. cit., 41; Canada Medical and Surgical Journal, I (1873): 170. Montreal Herald, 4 septembre 1868.
} 
cet organisme pan-canadien. Par contre, certains médecins pensèrent qu'un moyen de réformer la situation au Québec serait peut-être de mettre sur pied un bureau, relevant du Collège, qui pourrait vérifier la qualité de la formation donnée aux étudiants et le sérieux des examens. L'idée fera d'ailleurs surface à nouveau plus tard.

Au Québec, durant les années 1870, les groupes qui militèrent avec le plus de force afin de donner davantage d'influence au Collège au sujet de la question des licences, furent ceux de la Société médicale de Montréal et de l'Union médicale du Canada ${ }^{40}$. D'ailleurs, certaines personnes militaient au sein des deux organismes, tels J.-P. Rottot, E.P. Lachapelle, G. Grenier, A. Dagenais, A. Lamarche. Trois d'entre eux enseignaient aussi à l'École de médecine de Montréal. Leurs objectifs étaient clairs de même que les motifs qui les guidaient.

Actuellement les Universités sont toutes puissantes, non seulement elles se créent des élèves, mais encore, au terme d'un cours de médecine dont elles déterminent, sans contrôle, la nature et la durée, elles octroient des diplômes qui donnent au porteur le droit d'exercer la médecine. Le Collège des médecins et chirurgiens, qui est l'administrateur de la loi du pays, devrait posséder le pouvoir de s'assurer de la manière la plus complète possible des qualifications et des connaissances de ceux qui entreprennent les devoirs si importants de médecin, chirurgien et accoucheur. (...) On se plaint partout de l'encombrement de la profession; d'où provientil, sinon de la facilité avec laquelle on admet aujourd'hui les aspirants à l'étude et à la pratique de la médecine; (...) Le meilleur moyen d'obtenir ce résultat c'est de suivre l'exemple des autres professions, c'est-à-dire d'enlever aux Universités les privilèges exorbitants qu'elles possèdent aujourd'hui. ${ }^{41}$

Ils soumirent en ce sens un projet de loi qui fut étudié par un Comité spécial de la Chambre, composé de douze personnes dont huit médecins ${ }^{42}$. Plusieurs amendements furent apportés au projet de loi initial; on n'était pas d'accord pour «enlever aux universités leurs privilèges». Afin d'essayer de satisfaire le plus grand nombre, on se rabattit sur l'idée des «visiteurs» avancée en 1868. La loi votée en 1876 reconnaissait aux universités le privilège de donner des diplômes ad practicandum, mais celles-ci étaient désormais obligées de soumettre leurs examens à la surveillance d' "assesseurs» nommés par le Bureau du Collège. La tâche de ces évaluateurs consistait à assister aux examens médicaux des diverses universités et à faire rapport au Bureau sur la nature des examens. Dans le cas de rapports défavorables, le Bureau pouvait refuser d'enregistrer les diplômes de ces institutions.

\footnotetext{
40 L'Union médicale du Canada, VII, 2 (février 1877): 83.

41 L'Union médicale du Canada, IV, 11 (novembre 1875): 522. Les italiques sont de nous.

42 L'Union médicale du Canada, VII, 2 (février 1877): 82.
} 
L'établissement de ce droit de surveillance représentait une victoire, bien que partielle, pour le Collège. Celui-ci n'obtenait pas, comme il le voulait, le contrôle exclusif des examens de fin d'études, mais au moins on lui reconnaissait un droit de regard sur ces examens. L'éditorialiste de l'Union médicale du Canada (Rottot), qui était aussi le nouveau président du Collège, commentait ainsi cette loi:

Un bureau central d'examen aurait certainement donné des résultats plus satisfaisants, mais, pour l'avenir, il aurait fallu entreprendre une lutte avec les universités et courir le risque de ne rien avoir du tout, pour avoir voulu tout avoir. Aussi, croyons-nous que, vu ces circonstances et l'heure avancée de la session, il était plus sage de céder quelque chose et d'accepter un compromis qui, tout en sanctionnant le principe de notre prétention, nous assurait une nouvelle législation qu'il nous sera toujours plus facile de compléter plus tard. ${ }^{43}$

Le Collège ne voulut pas rester sur ce demi-échec. En 1886 un comité du Collège composé des docteurs Lemieux, G. Ross, Hingston, Campbell, E.-P. Lachapelle, Rodgers, Belleau et de Saint-George soumit aux médecins réunis à l'assemblée triennale l'idée de la création d'un Bureau central d'examinateurs relevant du Collège. Le projet fut accepté mais il ne parvint pas à la Chambre des députés parce que vraisemblablement, on n'arriva pas, par la suite, à s'entendre sur sa composition. Ce n'est que beaucoup plus tard, en 1909, qu'il refit surface. Cette année-là effectivement, peut-être pour contrecarrer la menace que représentait à leurs yeux le projet Roddick, ou pour faire comme les avocats et les notaires qui avaient déjà chacun leur bureau central d'examinateurs, on fit beaucoup d'efforts pour en arriver à un compromis. On s'entendit pour créer ce qu'on appela un Bureau provincial d'examinateurs qui relèverait de la juridiction du Collège, mais qui serait composé, pour les deux tiers, des professeurs des facultés de médecine des universités et pour un tiers des représentants du Collège ${ }^{44}$.

Désormais, tous les aspirants à la pratique seraient soumis aux mêmes examens. La lutte avait été longue et difficile, il avait fallu 62 années pour en arriver là.

\section{Conclusion}

On voit donc que ce projet d'encadrement et de standardisation des études médicales a donné des résultats. Au tournant du $\mathrm{XX}^{\mathrm{e}}$ siècle, tous les étudiants en médecine du Québec fréquentent une école de médecine et obtiennent la même formation. Seuls peuvent entreprendre des études médicales ceux qui ont fait des études classiques ou l'équi-

43 L'Union médicale du Canada, VII, 2 (février 1877): 81.

44 Statuts de la Province de Québec, 9 Edward VII, chap. 55 (1909). 
valent; tous reçoivent, par le biais des facultés de médecine les mêmes cours, et tous sont soumis aux mêmes examens au terme de ces études.

Ce processus d'uniformisation s'est fait lentement. Il a commencé dès 1847 en exigeant une formation classique de base de la part des aspirants aux études médicales. Cette loi obligea également ceux qui étaient admis en médecine à fréquenter une institution de formation reconnue et à suivre un cursus obligatoire sur le plan théorique et pratique.

La loi de 1876 visait à mettre fin à de nombreux mécontentements exprimés surtout depuis le milieu des années 1860. Elle marqua un tournant en ce sens qu'elle vint uniformiser un certain nombre de pratiques différentes au sein des universités, et reconnaître au Collège un rôle de surveillance plus grand en ce qui avait trait aux examens d'entrée, aux études et aux examens terminaux. Ce rôle sera à nouveau confirmé et renforcé en 1909.

Un tel encadrement de la médecine et des étudiants mettait évidemment en cause des intérêts majeurs. Le contrôle des admissions à l'étude et à la pratique permit bien sûr de hausser la qualité des études, mais aussi de limiter le nombre des nouveaux admis à une époque où on se plaignait d'un surplus de praticiens ${ }^{45}$. Par ailleurs, la mise en place d'un cursus médical plus poussé, mais exclusif (le seul reconnu) et obligatoire pour tous, permit la consolidation de la médecine professionnelle à une époque où ses assises n'étaient pas encore solides. Grâce à cette réforme des études médicales, les diplômés en médecine des universités purent non seulement s'imposer, par leurs études, comme les mieux formés et les plus compétents, mais aussi comme étant les seuls à pouvoir être autorisés à pratiquer la médecine dans la province. M.S. Larson commenta ainsi ce phénomène qu'elle observait aussi chez d'autres professions en Angleterre et aux États-Unis:

Only with the rise of formal training institutions and standardized training can professions begin to assume that there is a commonality among their members. (...) Cognitive standardization appears to be one crucial, if not the most crucial, variable in the sequence which, passing through the rise of monopolistic centers of training, leads to credentialed professionalisation and market control. ${ }^{46}$

Comme on l'a vu, le tournant décisif de cette lutte entre le Collège et les facultés de médecine eut lieu au milieu des années 1870. Il est intéressant de remarquer que cette intervention du Collège dans le domaine des études médicales coïncide avec les démarches qu'il fait en

\footnotetext{
45 J. Bernier, «Les praticiens de la santé au Québec, 1871-1921»; quelques données statistiques, Recherches socigraphiques, XX, 1 (janvier-avril 1979): 41-58.

46 M.S. Larson, The Rise of Professionalism, a Sociological Analysis (Berkeley, University of California Press, 1977), 46. Les italiques sont de nous.
} 
vue de renforcer son influence auprès des praticiens en rendant l'enregistrement au Collège obligatoire et en leur imposant un premier code d'éthique médicale. Ce projet fut, en grande partie, l'oeuvre d'un groupe assez homogène de médecins de Montréal, qui étaient actifs au sein de la Société médicale de Montréal et de l'Union médicale du Canada fondée en 1872. C'est d'ailleurs le porte-parole le plus en vue de ce groupe, J.-P. Rottot, qui fut élu à la présidence du Collège en 1877. 